\title{
Bandpass integrated Bragg gratings in silicon-on- insulator with well-controlled amplitude and phase responses
}

\author{
Alexandre D. Simard, ${ }^{1}$ Michael J. Strain, ${ }^{2,3}$ Laura Meriggi, ${ }^{2}$ Marc Sorel, ${ }^{2}$ and Sophie LaRochelle ${ }^{1, *}$ \\ ${ }^{1}$ COPL, Department of Electrical and Computer Engineering, Université Laval, Québec, QC, G1V OA6, Canada \\ ${ }^{2}$ School of Engineering, University of Glasgow, Glasgow, G12 8LT, U. K. \\ ${ }^{3}$ now with the Institute of Photonics, University of Strathclyde, Glasgow, G4 ONW, U.K. \\ *sophie.larochelle@gel.ulaval.ca \\ Received Month X, XXXX; revised Month X, XXXX; accepted Month X, \\ XXXX; posted Month X, XXXX (Doc. ID XXXXX); published Month X, XXXX

\begin{abstract}
Bandpass filters with square shape amplitude responses and well-controlled dispersion characteristics are achieved by accurate apodization of Bragg grating structures in silicon-on-insulator waveguides. For these devices, precise tailoring of their frequency response typically requires low coupling coefficients and relatively long on-chip propagation lengths. These challenges are addressed by implementing apodization by phase-modulation and using wider strip waveguides to reduce phase noise. This design approach is demonstrated with a dispersion-less narrowband filter and a chirped bandpass filter. (C) 2014 Optical Society of America

OCIS Codes: (050.2770) Gratings, (130.3120) Integrated optics devices, (130.7408) Wavelength filtering devices, (140.4780) Optical resonators.
\end{abstract}

Fiber Bragg gratings have been used in a wide variety of applications, such as pulse shaping [1], multichannel dispersion compensation [2], add/drop filter [3,4]. Recently, much effort has been devoted to implement these devices in silicon-on-insulator (SOI) using integrated Bragg gratings (IBGs) in strip or rib waveguides. Uniform [5,6], phase shifted [5,7], superimposed [8] and chirped gratings [9,10] have already been demonstrated. However, to obtain high quality bandpass filters with well-controlled phase responses, it is necessary to fabricate longer IBGs with weaker coupling coefficients. In this paper, we discuss in detail the challenging aspects of designing and implementing bandpass filters with precisely tailored amplitude and phase responses using IBGs in SOI.

In nanowire SOI waveguides with sidewall gratings, the strong overlap of the optical mode with the grating perturbation leads to large coupling coefficients $(\kappa)$, typically $\kappa>100 \mathrm{~cm}^{-1}$ even when the grating corrugation amplitudes are limited to a few nanometers. In order to reduce $\kappa$ and obtain narrowband filters, waveguide geometries such as rib waveguides can be considered [6]. Nevertheless, when apodization of these weak gratings becomes necessary to shape the filter passband, this approach remains limited by the resolution of the fabrication process. Alternative apodization techniques, for example based on a modification of the corrugation duty-cycle, have also been proposed [11]. However, in addition to the variation of the local coupling coefficient, these approaches also involve a significant modification of the local effective index, which must be accurately known and compensated in order to avoid distortions in the grating spectral response. For weakly coupled gratings, the dimensions of these corrections fall below the resolution limit of most fabrication processes.

Recently, we proposed that phase-modulation could be used to apodize IBGs in SOI and we demonstrated the implementation of a simple Gaussian apodization profile in a third-order grating using a CMOS compatible fabrication process [12]. In this paper, we show that complex apodization profiles of narrowband dispersionless filter and of a chirped bandpass filter, can be precisely tailored and successfully implemented with this phasemodulation apodization technique.

The phase-modulation apodization method maintains constant corrugation amplitude along the grating length at a value set by the desired maximal coupling coefficient. By varying the position of the corrugation, or equivalently by modulating the grating period, the local effective grating coupling coefficient, is modified thereby producing an apodization profile, $\kappa(z)$ [12]. This apodization method is very robust to fabrication errors as the corrugation amplitude is not changed along the propagation axis and, therefore, fabrication errors such as over- or underetching will have similar effect over the whole grating length. As a result only an average offset in the Bragg wavelength, and a small scaling of the coupling coefficient, are introduced but distortions of the spectral response is negligible. Furthermore, very low coupling coefficients can be obtained, which is important to achieve a complete apodization profile rather than a truncated one.

As an example, in this paper, we examine two IBG filters with a square shape amplitude response over a bandwidth of only a few nanometers. The first design has an in-band dispersion-less phase response, which is of interest for chip-scale wavelength-division multiplexing (WDM) networks, and the second has a linear group delay, which is of interest for on-chip phase engineering. Devices similar to the second design (chirped filter) have already been fabricated in SOI [9,10], however with bandwidths ranging from $20 \mathrm{~nm}$ to $100 \mathrm{~nm}$ and relatively low side-lobe suppression ratio (SLSR) (from 4 to $8 \mathrm{~dB}$ ). The first design (dispersion-less filter) has never been fabricated in SOI likely due to its high phase noise sensitivity. 
Fig. 1 shows the amplitude and phase profiles of the target spectral responses of both designs: dispersion-less filter (green) and chirped filter (blue). From these spectral characteristics, we use an integral layer peeling (ILP) algorithm to perform the grating design [13]. The ILP algorithm provides a grating structure that is arbitrary long and thus must be truncated. The spectral response of the designed grating is then calculated by a standard transfer matrix solution of the coupled mode equations. Results are also shown in Fig. 1 for the dispersion-less filter (black) and chirped filter (red). As can be seen in Fig. 1(a), the truncation introduces a noise floor, in this case below $-60 \mathrm{~dB}$. As can be seen in Fig. 1(a), both designs have different bandwidth. This design choice has been made to obtain two gratings with identical length, hence having similar phase noise impairments. The designed grating profiles are shown in Fig. 2(a) and Fig. 3(a)-(b). Fig. 2(a) shows the apodization profile of the grating coupling coefficient (grating strength) for the dispersionless filter. The small coupling coefficient amplitude on each sides of the main lobe clearly indicates that a very reliable apodization technique is required to fabricate this grating. The grating phase profile (not shown) is uniform with $\Pi$-phase shifts at the positions where the coupling coefficient goes to zero. Fig. 3(a) and Fig. 3(b) show respectively the phase and amplitude profiles of the chirped grating where it can be seen that non-symmetric profiles are required to obtain a linear phase response.

Using the proposed approach, the complexity of the grating apodization profile is completely put into the grating phase by adding a slow modulation function with a $z$ dependant amplitude. The effective index of the

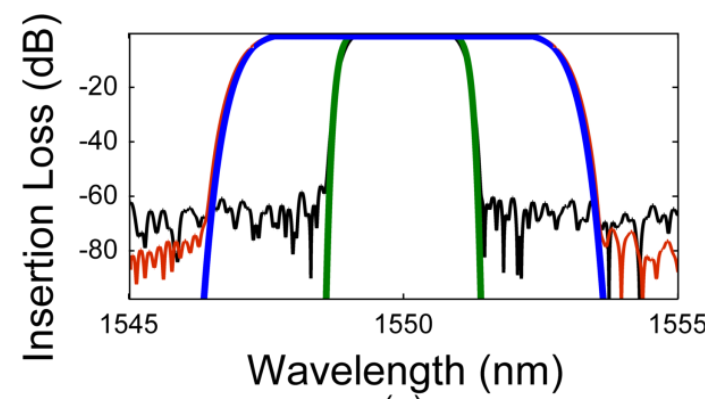

(a)

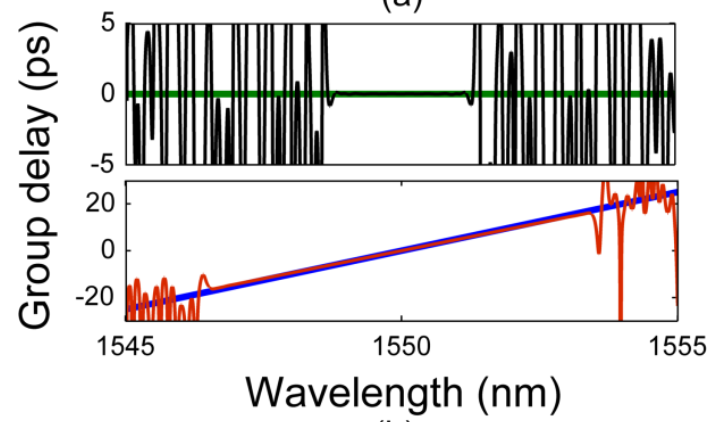

(b)

Fig. 1. (a) Amplitude and (b) group delay response of the target dispersion-less filter (green), the target $5 \mathrm{ps} / \mathrm{nm}$ dispersion filter (blue), the designed dispersion-less filter obtained by integral layer peeling (black) and the designed $5 \mathrm{ps} / \mathrm{nm}$ dispersion filter, also obtained by integral layer peeling, (red). grating can be expressed as

$$
n=n_{0}(\lambda)+\Delta n \cos \left(\frac{2 \pi}{\Lambda} z+\theta(z)+\phi(z) \sin \left(\frac{2 \pi z}{\Lambda_{M}}\right)\right) .
$$

By expanding this equation with Fourier series, equation (1) becomes

$$
n=n_{0}(\lambda)+\Delta n \sum_{m=-\infty}^{\infty} J_{m}(\phi(z)) \cos \left(\frac{2 \pi}{\Lambda} z+m \frac{2 \pi}{\Lambda_{M}} z+\theta(z)\right) .
$$

The modulation period $\left(\Lambda_{M}\right)$ must therefore be chosen small enough to prevent perturbation of the desired grating resonance $(m=0)$ by echos $(m \neq 0)$ located at different resonant frequencies. Of course $\Lambda_{M}$ must still be compatible with the fabrication process. The required $\Lambda_{M}$ can be determined from the desired spectral spacing between the main resonance and the first echo $(\Delta \mathrm{d})$ using

$$
\Lambda_{M}=\frac{\bar{\lambda}_{B}}{2 n_{g}}\left(\frac{\bar{\lambda}_{B}}{\Delta \lambda}-1\right)
$$

where $\bar{\lambda}_{B}$ is the average Bragg wavelength and $n_{g}$ is the group index. In this work, $\Delta \lambda$ has been fixed at $20 \mathrm{~nm}$ and $\Lambda_{M}=15 \mu \mathrm{m}$. From eq. (1) and eq. (2), to apodize the grating, the phase term, $\phi(z)$, must be equal to $J_{0}^{-1}(f(z))$, where $f(z)$ is the target normalized apodization profile.

Fig. 2(b) shows the phase apodization profile expressed in terms of the local Bragg wavelength and Fig. 3(c) also shows this profile compared to the original Bragg wavelength profile of Fig. 3(a). This phase modulated profile is converted into a physical grating period profile, which is converted in a computer-aided design (CAD) layout using an open-source GDS Matlab library [14].

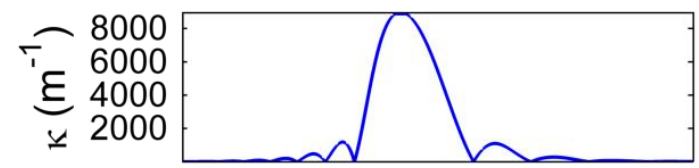

(a)
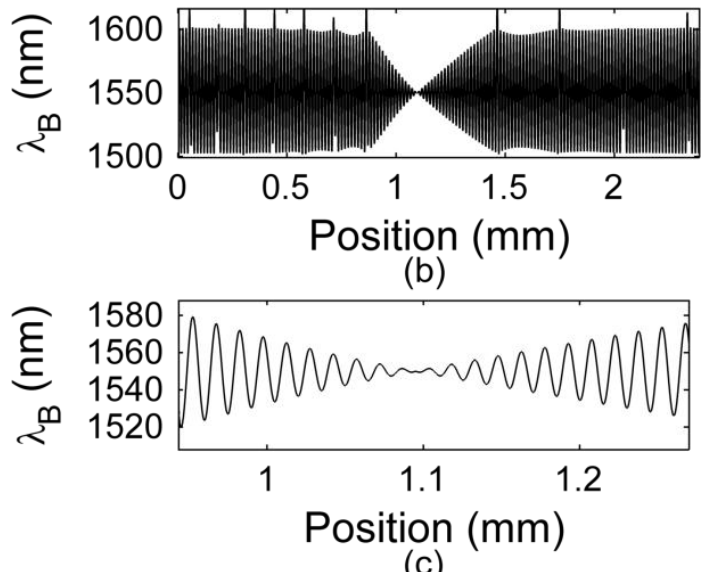

(c)

Fig. 2. (a) Coupling coefficient profile of the dispersion-less square-like IBGs shown in Fig. 1 (black). (b) Bragg wavelength profile that contains the apodization information. (c) Zoom on the central section of (b). 


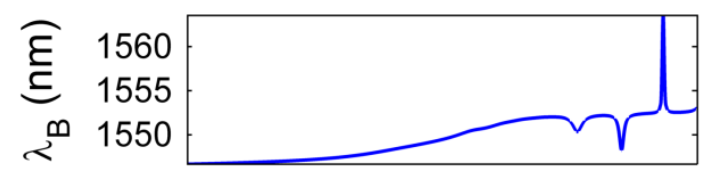

(a)

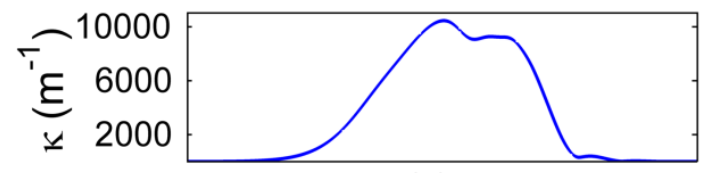

(b)

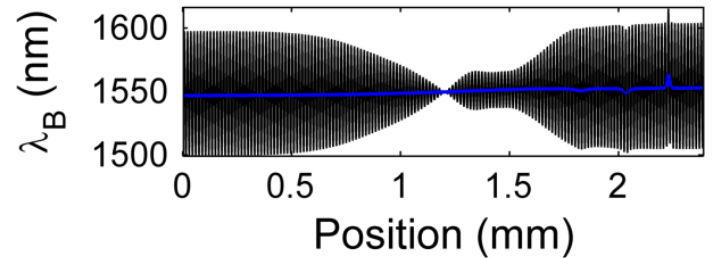

(c)

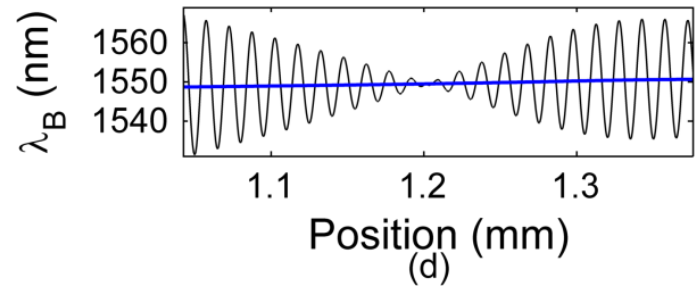

Fig. 3. (a) Bragg wavelength and (b) coupling coefficient profiles of the $5 \mathrm{ps} / \mathrm{nm}$ dispersion square-like IBGs shown in Fig. 1 (red). (c) Bragg wavelength profile that contains the apodization information (in black) compared to the one shown in (a). (d) Zoom on the centeral section of (c).

The relatively long length and weak coupling coefficient of these IBGs $(2.5 \mathrm{~mm})$ make them susceptible to spectral distortions introduced by accumulated phase noise $[15,16]$. Phase noise due to sidewall roughness is known to be an important limitation for long IBGs in singlemode waveguides due to the strong mode field and high index contrast at the core boundary. This phase noise can be markedly reduced by using wider multimode waveguides or rib waveguides. The use of multimode waveguides is not problematic provided that only the fundamental mode is excited.

The designs of Fig. 2 and Fig. 3 were fabricated in waveguides with widths of $1000 \mathrm{~nm}$ and $800 \mathrm{~nm}$ respectively. IBG were introduced by placing corrugations on both waveguide sidewalls. The corrugation amplitudes, determined with FDTD simulations, were respectively 50 $\mathrm{nm}$ and $40 \mathrm{~nm}$. All the devices are fabricated in SOI with a $220 \mathrm{~nm}$ core and $2 \mu \mathrm{m}$ buried oxide undercladding at the Jame Watt Nonofabrication Center (University of Glasgow). The waveguides and gratings were written using electron-beam lithography into negative tone hydrogen silsesquioxane (HSQ) resist, which then acted as a hard mask for the ICP-RIE etching of the silicon material. The resolution grid of the 100kV Leica VB6 tool used for the lithography was $1 \mathrm{~nm}$ and the beam spot was set at $4 \mathrm{~nm}$. The waveguides were terminated in inverse tapers and SU8 mode converter waveguides in order to minimize facet reflectivities and coupling losses to fiber, which were measure to be about $5 \mathrm{~dB}$. A waveguide length of $500 \mu \mathrm{m}$, without corrugation, was present between the inverse taper and the IBGs. The devices were finally coated with an uppercladding of HSQ and plasma enhanced chemical vapor deposition (PECVD) silica. The complex spectral responses were measured with a commercial optical frequency domain reflectometer, to which we removed the coupling losses. To fully eliminate the reflections at the chip input/output, we performed time filtering of the grating impulse response [17].

Fig. 4 and Fig. 5 show the spectral response measured (blue) and calculated (black) from the apodized grating designs described in Fig. 2 and Fig. 3. Typical criteria to evaluate the sharpness of the grating amplitude are the 3 $\mathrm{dB}$ bandwidth (BW), the $10 \mathrm{~dB} \mathrm{BW}$ and the rising $\mathrm{BW}$ (BW over which the reflectivity goes from $10 \%$ to $90 \%$ ). These parameters, estimated from the design simulations and experimental measurements, are included in Table 1.

As illustrated in Fig. 4(a), the dispersion-less filter is in good agreement with the design and very small degradation of the bandwidth parameters is observed. Moreover, the SLSR is better than $10 \mathrm{~dB}$, which is very good result for long IBGs in SOI $(>1 \mathrm{~mm})$ due to the difficulty to reproduce the tails of the apodization profile where the coupling coefficient is very low, and due to the accumulated phase noise [15]. Similarly, as shown in Fig. $5(\mathrm{a})$, the square-shaped grating having a $5 \mathrm{ps} / \mathrm{nm}$ dispersion follows well the design although the $3 \mathrm{~dB}$ BW and $10 \mathrm{~dB}$ BW are slightly larger, but the grating rising BW is still very close to the design. Finally, as shown in Table 1, the measured grating reflectivity indicates that the coupling coefficient is slightly larger than the design by about $10 \%$.

Fig. 4(b) and Fig. 5(b) show the experimentally measured group delay for both filters. As for the amplitude, the phase response follows the design closely. For the dispersion-less IBG, the group delay ripples have a standard deviation of $2.3 \mathrm{ps}$ and for the $5 \mathrm{ps} / \mathrm{nm}$ dispersion grating, the standard deviation from a $5 \mathrm{ps} / \mathrm{nm}$ linear group delay (i.e. the black curve) is very small at 1.5 ps. The spectral range displayed in Fig. 4 and Fig. 5 is relatively narrow to allow a better comparison between the design and the experimental responses. Fig. 6 shows the experimental response of the dispersion-less grating over a wider spectral range. The $5 \mathrm{ps} / \mathrm{nm}$ dispersion grating response is not presented but shows a similar

Table 1. Grating design performances

\begin{tabular}{|cc|c|c|c|c|c|}
\hline Gratings & & $\mathbf{k}_{\max }$ & $\left|\mathbf{r}_{\max }\right|^{2}$ & $\begin{array}{c}3 \mathbf{d B} \text { BW } \\
{[\mathbf{n m}]}\end{array}$ & $\begin{array}{c}10 \mathrm{~dB} \text { BW } \\
{[\mathbf{n m}]}\end{array}$ & $\begin{array}{c}\text { Rising BW } \\
{[\mathbf{n m}]}\end{array}$ \\
\hline $\begin{array}{c}\text { Square-shaped dispersion-less } \\
\text { grating (design shown in Fig. 2) }\end{array}$ & Design & $9000 \mathrm{~m}^{-1} \cdot$ & 0.99 & 2 & 2.2 & 0.36 \\
\hline $\begin{array}{c}\text { Square-shaped 5 ps/nm dispersion } \\
\text { grating (design shown in Fig. 3) }\end{array}$ & Experiment & $\sim 10000 \mathrm{~m}^{-1}$. & $\sim 0.99-0.995$ & 2.32 & 2.6 & $0.42 / 0.48$ \\
\cline { 2 - 8 } & Experiment & $10500 \mathrm{~m}^{-1}$. & 0.99 & 5 & 5.9 & 0.78 \\
\hline
\end{tabular}




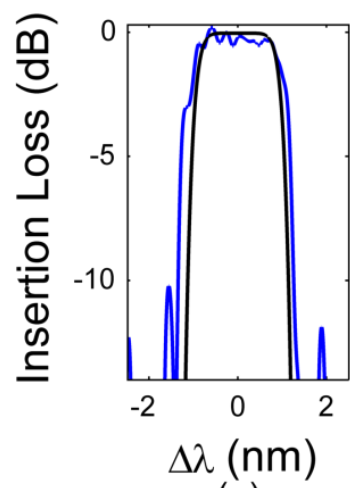

(a)

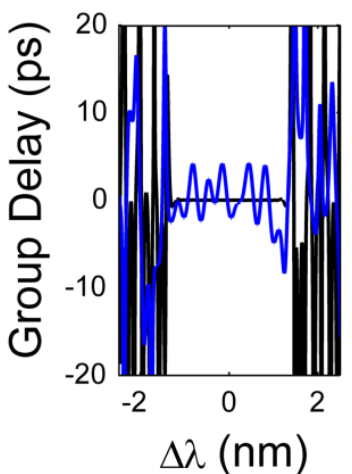

(b)
Fig. 4. (a) Spectral response and (b) group delay of a squareshaped dispersion-less grating in reflection. The blue curve is the experimental response and the black is the design. The IBG has a central wavelength at $1564.5 \mathrm{~nm}$

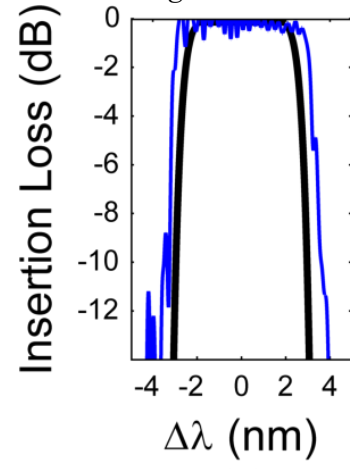

(a)

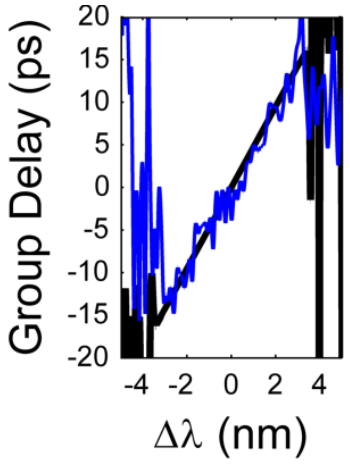

(b)
Fig. 5. (a) Spectral response and (b) group delay of a squareshaped $5 \mathrm{ps} / \mathrm{nm}$ dispersion grating in reflection. The blue curve is the experimental response, and the black is the design. The IBG has a central wavelength at $1569 \mathrm{~nm}$

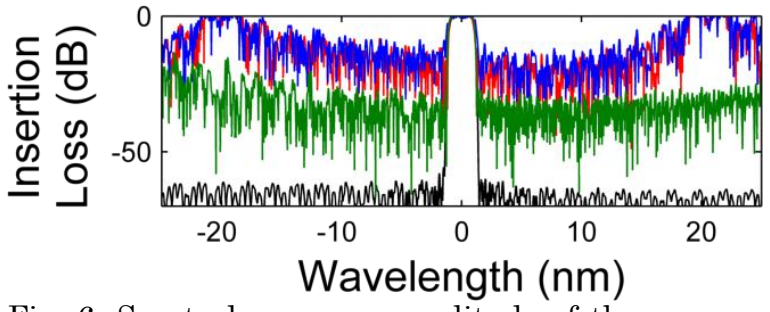

Fig. 6. Spectral response amplitude of the square-shaped dispersion-less grating Fig. 4 showing the experimentally measured response in reflection (blue), the calculated response from the ideal apodized grating design (black) and the simulated response of the phase-apodized grating $\left(\Lambda_{M}=15 \mu \mathrm{m}\right.$, red $)$. The green curve shows the simulation for a smaller modulation period $\left(\Lambda_{\mathrm{M}}=6 \mu \mathrm{m}\right)$

behavior. As can be seen from Fig. 6, the noise floor of the measured IBG (in blue) is significantly higher than the grating response simulated with an ideal apodization profile (in black). This noise floor of approximately $-15 \mathrm{~dB}$ comes from the phase apodization. As can be seen on this figure, the echoes, located on both sides of the main peak, have tails that extend to below the grating main lobe. This noise floor is also predicted by simulation of the grating response simulated with an apodization profile implemented through phase modulation (in red) (i.e. with the profile shown in Fig. 2(b)). Future work will consider using a smaller modulation period to move these echoes farther from the main grating resonances, which should reduce the noise floor and increase the SLSR as shown by the green curve of Fig. 6 calculated with $\Lambda_{\mathrm{M}}=6 \mu \mathrm{m}$. This new modulation period would slightly reduce the minimum feature size (from $150 \mathrm{~nm}$ to $120 \mathrm{~nm}$ ) but would not impact the corrugation amplitude.

In conclusion, we proposed and demonstrated a design approach to fabricate high quality IBGs bandpass filters in SOI. This method delivers square-shaped amplitude responses and well-controlled phase responses by using phase-modulation apodization and wider multimode waveguides to reduce the phase noise. Specifically, filters having sharp edges and low sidelobes, which are necessary for WDM networks and signal processing applications, have been fabricated. Compared to state-ofthe-art IBGs in SOI of similar length $(>1 \mathrm{~mm})$, the gratings presented in this paper have improved sidelobe suppression ratio due to the reduction of phase noise and more accurate apodization profiles. More importantly, this performance was achieved with a much narrower filtering bandwidth $(2 \mathrm{~nm}$ and $5 \mathrm{~nm})$, compared to previously reported devices that had bandwidth of few tens of nanometers. The technique could be further improved by optimizing the phase-modulation period in order to reduce the noise floor of the spectral response.

\section{References}

1. J. Azana, IEEE Photonics J. 2(3), 359-386 (2010).

2. Y. Painchaud, M. Lapointe, F. Trepanier, R. L. Lachance, C. Paquet, and M. Guy, in Opt. Fiber Commun. Conf. Opt. Soc. Am., 1-3 (2007).

3. I. Baumann, J. Seifert, W. Nowak, and M. Sauer, IEEE Photonics Technol. Lett. 8(10), 1331-1333 (1996).

4. M. N. Zervas and M. K. Durkin, Opt. Express 21(15), 17472-17483 (2013).

5. Y. Painchaud, M. Poulin, C. Latrasse, N. Ayotte, M.-J. Picard, and M. Morin, in Bragg Gratings Photosensit. Poling Glass Waveguides, BW2E.3, Optical Society of America (2012).

6. X. Wang, W. Shi, H. Yun, S. Grist, N. A. F. Jaeger, and L. Chrostowski, Opt. Express 20(14), 15547 (2012).

7. K. A. Rutkowska, D. Duchesne, M. J. Strain, R. Morandotti, M. Sorel, and J. Azaña, Opt. Express 19(20), 19514-19522 (2011).

8. M. J. Strain, S. Thoms, D. S. MacIntyre, and M. Sorel, Opt. Lett. 39(2), 413-416 (2014).

9. D. T. H. Tan, K. Ikeda, R. E. Saperstein, B. Slutsky, and Y. Fainman, Opt. Lett. 33(24), 3013-3015 (2008).

10. G. F. R. Chen, T. Wang, C. Donnelly, and D. T. H. Tan, Opt. Express 21(24), 29223-29230 (2013).

11. D. Wiesmann, C. David, R. Germann, D. Emi, and G. L. Bona, IEEE Photonics Technol Lett 12(6), 639-641 (2000).

12. A. D. Simard, N. Belhadj, Y. Painchaud, and S. LaRochelle, Photonics Technol. Lett. IEEE 24(12), 1033-1035 (2012).

13. A. Rosenthal and M. Horowitz, IEEE J. Quantum Electron. 39(8), 1018-1026 (2003).

14. N. Ayotte and A. D. Simard, <http://www.mathworks.com/ matlabcentral/fileexchange/file_infos/46827-nicolasayottematlabgdsphotonicstoolbox>.

15. A. D. Simard, N. Ayotte, Y. Painchaud, S. Bedard, and S. LaRochelle, J. Light. Technol. 29(24), 3693-3704 (2011).

16. A. D. Simard, G. Beaudin, V. Aimez, Y. Painchaud, and S. LaRochelle, Opt. Express 21(20), 23145-23159 (2013).

17. A. D. Simard, Y. Painchaud, and S. LaRochelle, in Bragg Gratings Photosensit. Poling Glass Waveguides, BM3D.7 (2012). 


\section{References}

1. J. Azana, "Ultrafast analog all-optical signal processors based on fiber-grating devices," IEEE Photonics J. 2(3), 359386 (2010).

2. Y. Painchaud, M. Lapointe, F. Trepanier, R. L. Lachance, C. Paquet, and M. Guy, "Recent progress on FBG-based tunable dispersion compensators for $40 \mathrm{~Gb} / \mathrm{s}$ applications," in Opt. Fiber Commun. Conf. Opt. Soc. Am., 1-3 (2007).

3. I. Baumann, J. Seifert, W. Nowak, and M. Sauer, "Compact all-fiber add-drop-multiplexer using fiber Bragg gratings," IEEE Photonics Technol. Lett. 8(10), 1331-1333 (1996).

4. M. N. Zervas and M. K. Durkin, "Physical insights into inverse-scattering profiles and symmetric dispersionless FBG designs," Opt. Express 21(15), 17472-17483 (2013).

5. Y. Painchaud, M. Poulin, C. Latrasse, N. Ayotte, M.-J. Picard, and M. Morin, "Bragg grating notch filters in siliconon-insulator waveguides," in Bragg Gratings Photosensit. Poling Glass Waveguides, BW2E.3, Optical Society of America (2012).

6. X. Wang, W. Shi, H. Yun, S. Grist, N. A. F. Jaeger, and L. Chrostowski, "Narrow-band waveguide Bragg gratings on SOI wafers with CMOS-compatible fabrication process," Opt. Express 20(14), 15547 (2012).

7. K. A. Rutkowska, D. Duchesne, M. J. Strain, R. Morandotti, M. Sorel, and J. Azaña, "Ultrafast all-optical temporal differentiators based on CMOS-compatible integratedwaveguide Bragg gratings," Opt. Express 19(20), 1951419522 (2011).

8. M. J. Strain, S. Thoms, D. S. MacIntyre, and M. Sorel, "Multi-wavelength filters in silicon using superposition sidewall Bragg grating devices," Opt. Lett. 39(2), 413-416 (2014).

9. D. T. H. Tan, K. Ikeda, R. E. Saperstein, B. Slutsky, and Y. Fainman, "Chip-scale dispersion engineering using chirped vertical gratings," Opt. Lett. 33(24), 3013-3015 (2008).

10. G. F. R. Chen, T. Wang, C. Donnelly, and D. T. H. Tan, "Second and third order dispersion generation using nonlinearly chirped silicon waveguide gratings," Opt. Express 21(24), 29223-29230 (2013).

11. D. Wiesmann, C. David, R. Germann, D. Emi, and G. L. Bona, "Apodized surface-corrugated gratings with varying duty cycles," IEEE Photonics Technol Lett 12(6), 639-641 (2000).

12. A. D. Simard, N. Belhadj, Y. Painchaud, and S. LaRochelle, "Apodized silicon-on-insulator Bragg gratings," Photonics Technol. Lett. IEEE 24(12), 1033-1035 (2012).

13. A. Rosenthal and M. Horowitz, "Inverse scattering algorithm for reconstructing strongly reflecting fiber Bragg gratings," IEEE J. Quantum Electron. 39(8), 1018-1026 (2003).

14. N. Ayotte and A. D. Simard, "Matlab GDS Photonics Toolbox," <http://www.mathworks.com/matlabcentral/ fileexchange/file_infos/46827-nicolasayottematlabgdsphotonicstoolbox $>$.

15. A. D. Simard, N. Ayotte, Y. Painchaud, S. Bedard, and S. LaRochelle, "Impact of sidewall roughness on integrated Bragg gratings," J. Light. Technol. 29(24), 3693-3704 (2011).

16. A. D. Simard, G. Beaudin, V. Aimez, Y. Painchaud, and S. LaRochelle, "Characterization and reduction of spectral distortions in silicon-on-insulator integrated Bragg gratings," Opt. Express 21(20), 23145-23159 (2013).

17. A. D. Simard, Y. Painchaud, and S. LaRochelle, "Characterization of integrated Bragg grating profiles," in Bragg Gratings Photosensit. Poling Glass Waveguides, BM3D.7 (2012). 